\title{
Editorial
}

\section{Control of Networked Systems with Engineering Applications}

\author{
Housheng Su, ${ }^{1}$ Michael Z. Q. Chen, ${ }^{2}$ Qing Hui, ${ }^{3}$ Yuan Fan, ${ }^{4}$ and Zhiwei Gao ${ }^{5}$ \\ ${ }^{1}$ School of Automation, Huazhong University of Science and Technology, Wuhan 430074, China \\ ${ }^{2}$ Department of Mechanical Engineering, The University of Hong Kong, Pokfulam Road, Pokfulam, Hong Kong \\ ${ }^{3}$ Department of Mechanical Engineering, Texas Tech University, Lubbock, TX, USA \\ ${ }^{4}$ School of Electrical and Electronic Engineering, Nanyang Technological University, Singapore \\ ${ }^{5}$ Biomedical Engineering, Northumbria University, Newcastle upon Tyne, UK \\ Correspondence should be addressed to Housheng Su; houshengsu@qq.com
}

Received 19 July 2016; Accepted 19 July 2016

Copyright (C) 2016 Housheng Su et al. This is an open access article distributed under the Creative Commons Attribution License, which permits unrestricted use, distribution, and reproduction in any medium, provided the original work is properly cited.

Recently, control of networked systems problems has attracted much attention among researchers in biology, physics, control engineering, and computer science for decades, partially due to the broad applications of control of networked systems in many engineering areas including cooperative control of mobile robots and estimation of mobile sensor networks. The study of complex network topology and modeling is to understand the influence of network structure on its function and consequently to find effective ways to improve network performance.

This special issue focuses on theoretical and technological achievements in control of networked systems. The contents of the accepted papers are summarized below.

(1) Coordinated control and controllability of networked systems: Y. Shi and J. Zhang ("Networked Convergence of Fractional-Order Multiagent Systems with a Leader and Delay") investigated the convergence of fractional-order discrete-time multiagent systems with a leader and sampling delay based on the Hermit-Biehle theorem. Z. Lu et al. ("Global Asymptotic Stability of Switched Neural Networks with Delays") presented the delay-dependent stability for a class of switched neural networks with timevarying delays via the quadratic convex combination. W. Liu et al. ("Robust Leaderless Consensus of Uncertain Multiagent Systems with Fast Switching Topologies") proposed a robust leaderless consensus algorithm of uncertain multiagent systems with directed fast switching topologies. B. Liu et al. ("Adaptive Second-Order Synchronization of Two Heterogeneous Nonlinear Coupled Networks") investigated the second-order synchronization of two heterogeneous nonlinear coupled networks by introducing adaptive control laws. L. Wang and X. Han ("Controllability of Multiagent Systems with a Directed Tree") studied the controllability of continuous-time networked systems based on the consensus protocol when the graph contains a directed spanning tree.

(2) Analysis and control of network structure: Z. Bogicevic et al. ("Graph-Analytical Method of Determining Impedance in Electrical Transformers") presented a graphanalytical method for determining the electrical impedance of alternate energy sources. R. Haghighi and H. Namazi ("Algorithm for Identifying Minimum Driver Nodes Based on Structural Controllability") investigated the structural controllability problem for networked systems, and a simple graph-based algorithm was presented to obtain the minimum driver nodes. A. Céspedes-Mota et al. ("Optimization of the Distribution and Localization of Wireless Sensor Networks Based on Differential Evolution Approach") studied the feasibility to apply the differential evolution algorithm in the presence of obstacles or strict restrictions. Y. Wei et al. ("Exploring the Impact of Network Structure and Demand Collaboration on the Dynamics of a Supply Chain Network Using a Robust Control Approach") studied the dynamics and robustness of a two-layer supply chain network of multiple retailers and multiple distributors. M. Ji et al. ("Semisupervised Community Detection by Voltage Drops") investigated a semisupervised community detection algorithm for partitioning network into groups. 
(3) Applications of control of networked systems: G. Yang and M. Yang ("Multistage Warning Indicators of Concrete Dam under Influences of Random Factors") proposed multistage warning indicators of concrete dam space and considered the influences of complex random factors. J.-H. Park et al. ("Adaptation Algorithm of Geometric Graphs for Robot Motion Planning in Dynamic Environments") presented an adaptive graph algorithm for collision-free motion planning of articulated robots in dynamic environments. Y. Chen et al. ("A Secure Network Coding Based on Broadcast Encryption in SDN") proposed a network coding to improve the capacity and robustness of multicast applications on software defined networks. X. Wu et al. ("A Swarm Intelligent Algorithm Based Route Maintaining Protocol for Mobile Sink Wireless Sensor Networks") investigated a novel route maintaining protocol based on the immune based artificial bee colony for the mobile sink wireless sensor networks. H. Zhang and X. Qi ("Heat Transfer Analysis and Modification of Thermal Probe for Gas-Solid Measurement") studied a gas-solid mass flow-rate measurement system and applied a novel modified thermal probe.

Note that although the selected topics and papers are not a comprehensive representation of the areas covered by the special issue, the published papers in this special issue do provide some recent advances in the field of control of networked systems, which could benefit the current research in some way.

\section{Acknowledgments}

We would like to thank all the authors for their contributions and acknowledge all the reviewers for their time and effort in assessing the manuscripts.

Housheng Su

Michael Z. Q. Chen

Qing Hui

Yuan Fan

Zhiwei Gao 


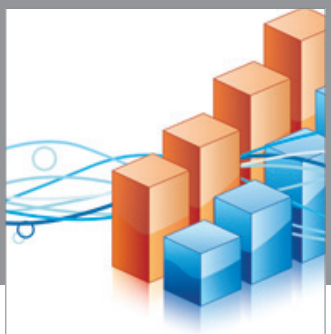

Advances in

Operations Research

vatem alat4

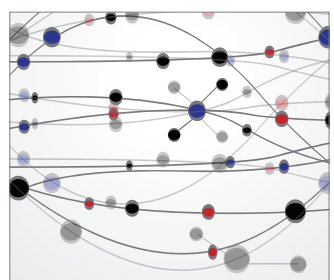

\section{The Scientific} World Journal
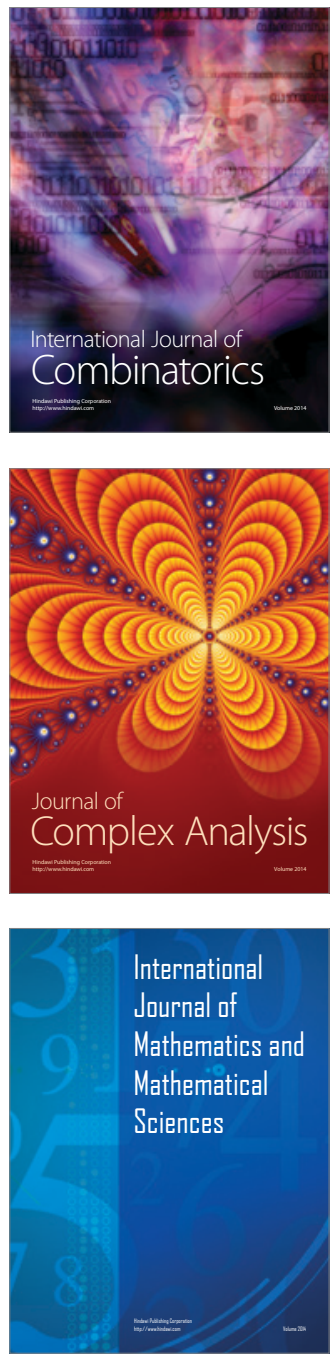
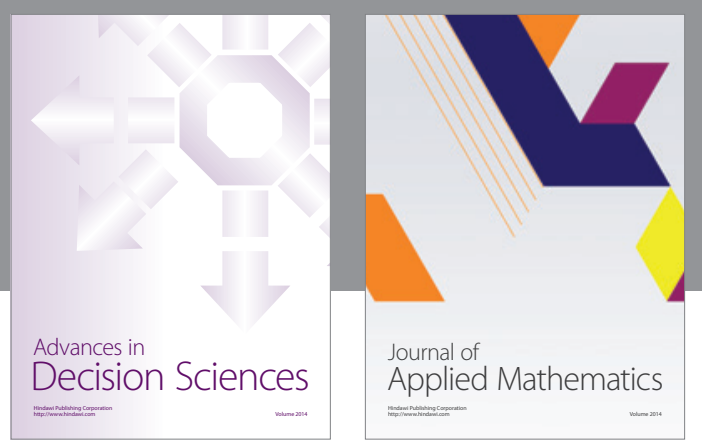

Algebra

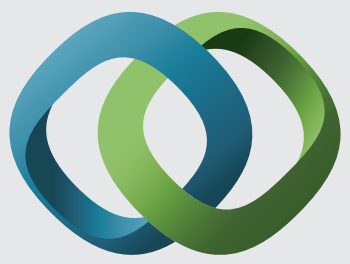

\section{Hindawi}

Submit your manuscripts at

http://www.hindawi.com
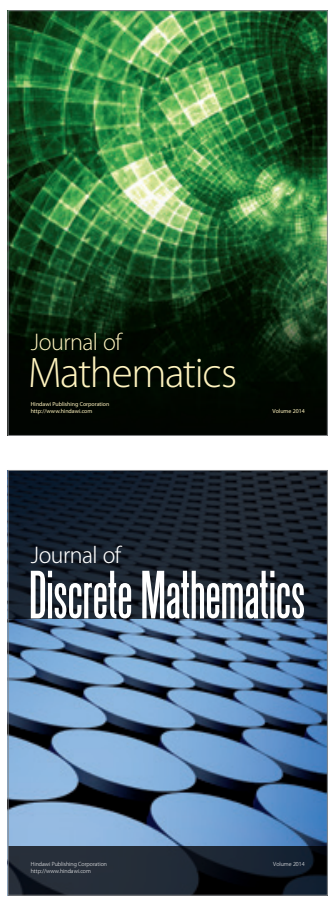

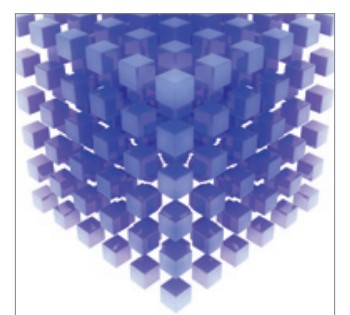

Mathematical Problems in Engineering
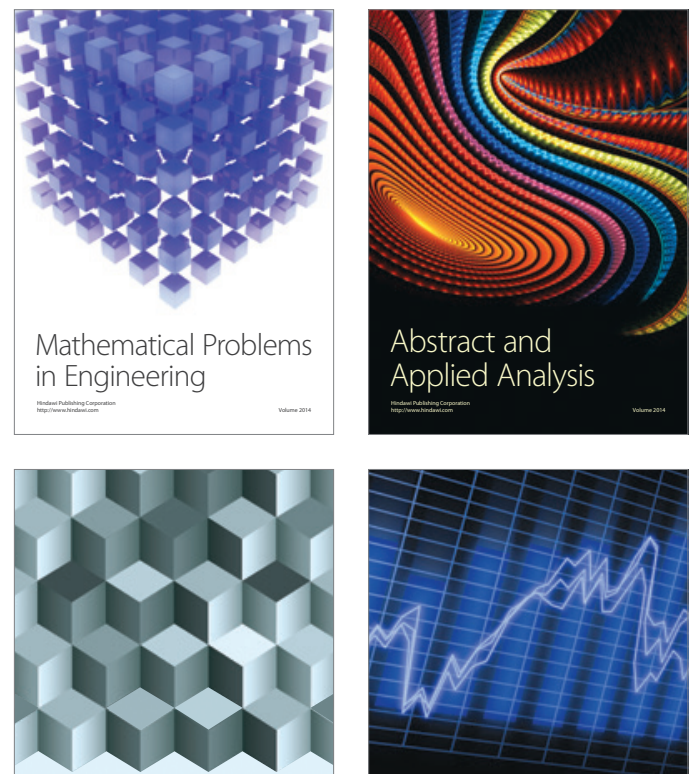

Journal of

Function Spaces

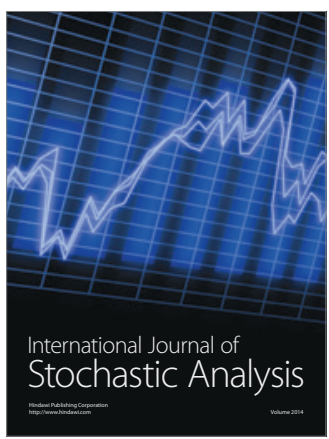

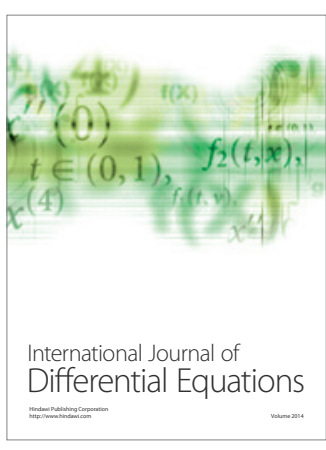
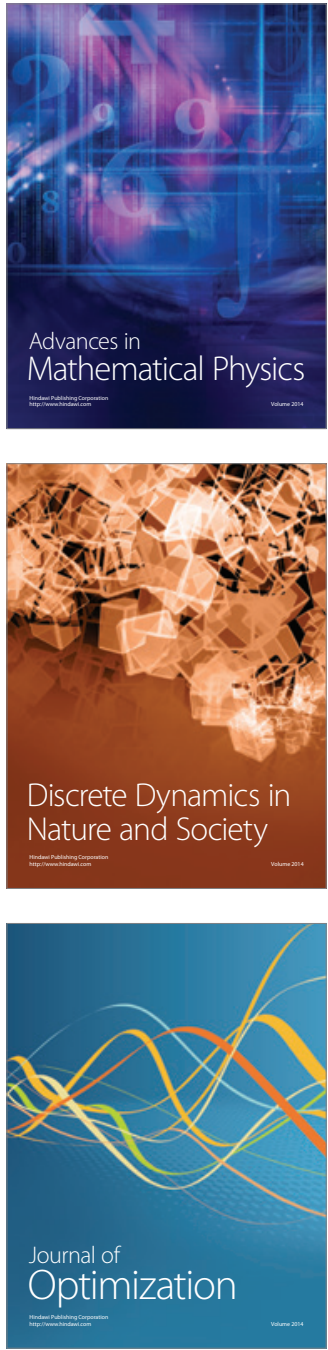